Erratum

\title{
Erratum to "Xiao-Xu-Ming Decoction Protects against Blood-Brain Barrier Disruption and Neurological Injury Induced by Cerebral Ischemia and Reperfusion in Rats"
}

\author{
Rui Lan, ${ }^{1}$ Jun Xiang, ${ }^{1,2}$ Guo-Hua Wang, ${ }^{1}$ Wen-Wei Li, ${ }^{1}$ Wen Zhang, \\ Li-Li Xu, ${ }^{1}$ and Ding-Fang Cai ${ }^{1}$ \\ ${ }^{1}$ Department of Integrative Medicine, Zhongshan Hospital, and Laboratory of Neurology, Institute of Integrative Medicine, \\ Fudan University, Shanghai 200032, China \\ ${ }^{2}$ Longhua Hospital, Shanghai University of Traditional Chinese Medicine, Shanghai 200032, China \\ Correspondence should be addressed to Ding-Fang Cai; dingfangcai@163.com \\ Received 19 June 2013; Accepted 27 August 2013 \\ Copyright (C) 2013 Rui Lan et al. This is an open access article distributed under the Creative Commons Attribution License, which \\ permits unrestricted use, distribution, and reproduction in any medium, provided the original work is properly cited.
}

The word "GADPH" should be "GAPDH" in line 23 of Section 2.10 and in Figure 6. 


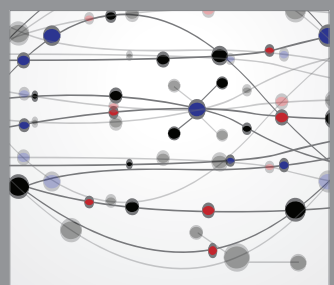

The Scientific World Journal
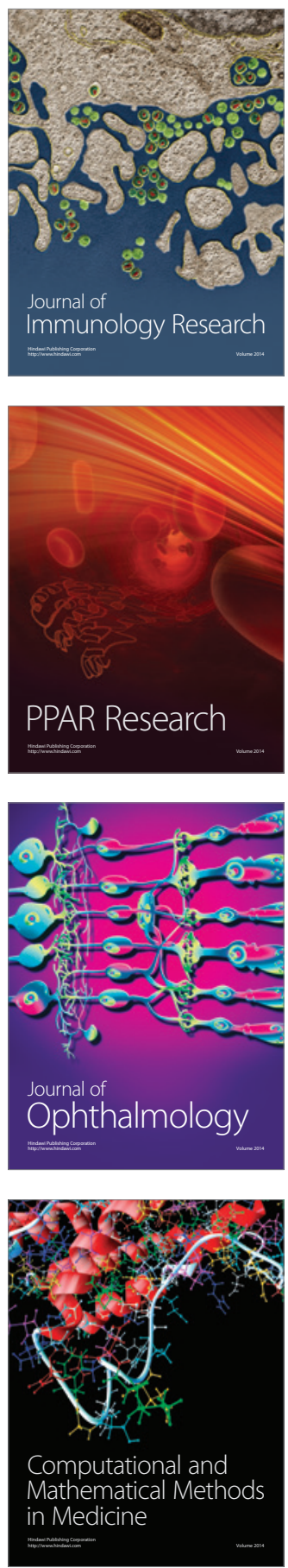

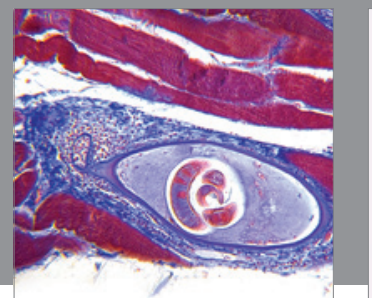

Gastroenterology

Research and Practice
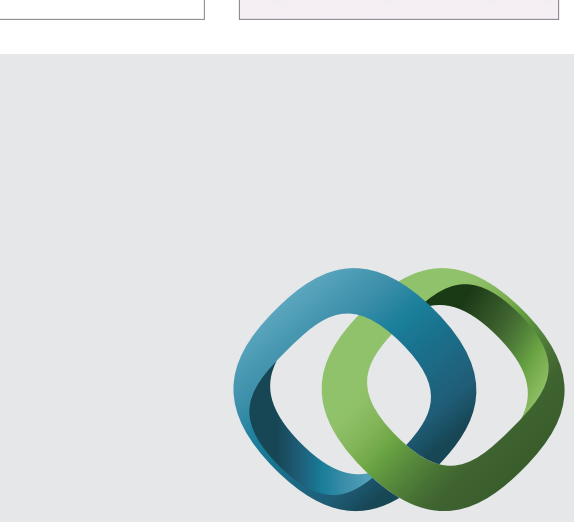

\section{Hindawi}

Submit your manuscripts at

http://www.hindawi.com
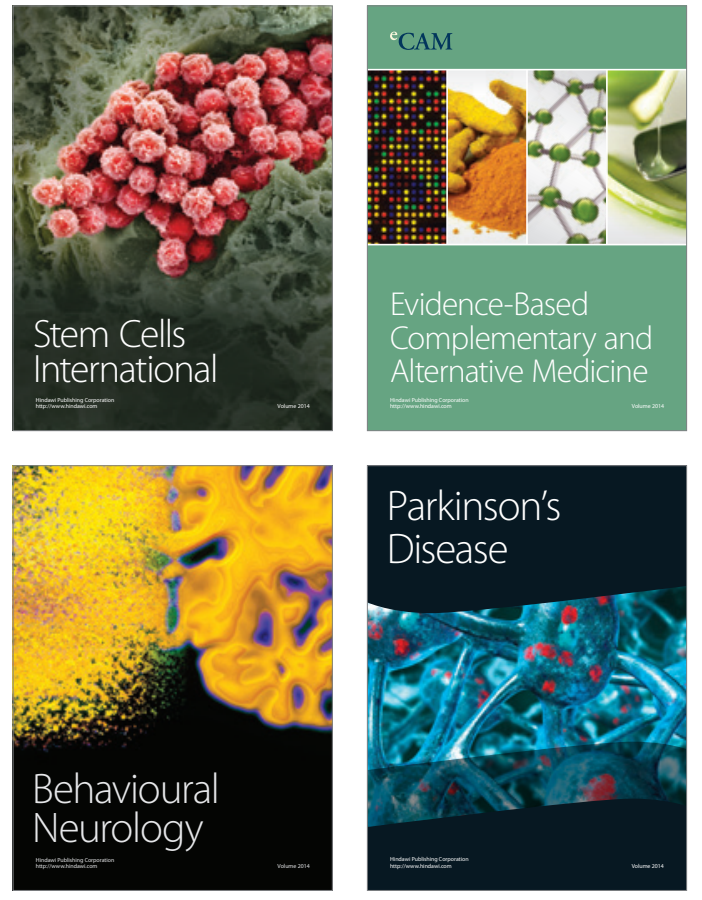
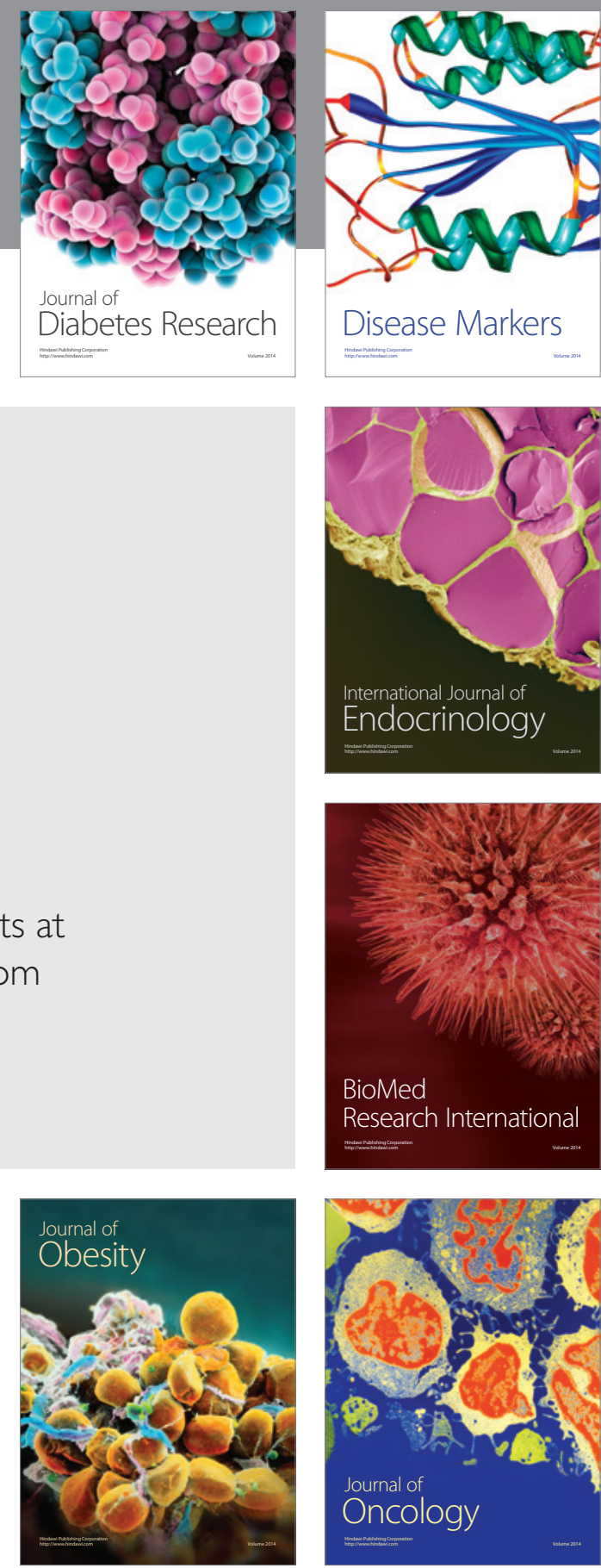

Disease Markers
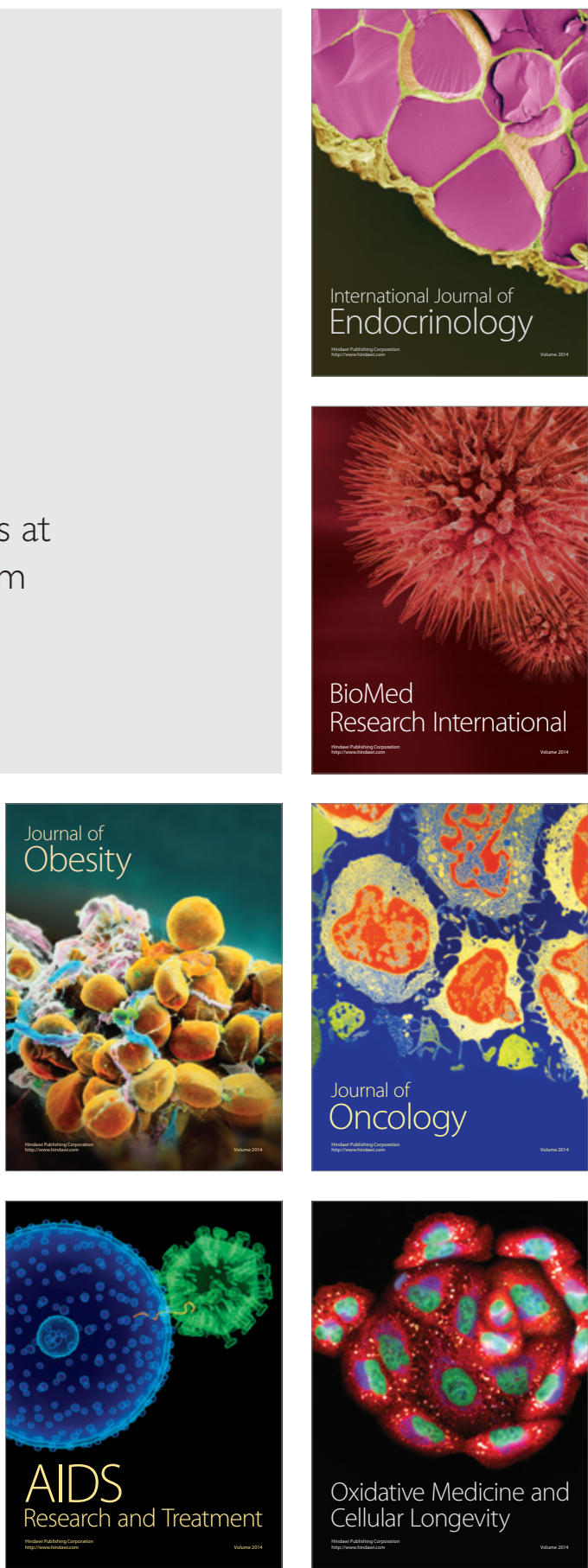